\title{
Graphical Notation for Diagramming Coupled Systems
}

\author{
J. Walter Larson ${ }^{1,2,3}$ \\ 1 Mathematics and Computer Science Division, Argonne National Laboratory, \\ Argonne, IL 60439, USA \\ larson@mcs.anl.gov \\ 2 Computation Institute, University of Chicago, Chicago, IL USA \\ 3 Department of Computer Science, The Australian National University \\ Canberra ACT 0200 Australia
}

\begin{abstract}
Multiphysics and multiscale-or coupled-systems share one fundamental requirement: Construction of coupling mechanisms to implement complex data exchanges between a system's constituent models. I have created a graphical schema for describing coupling workflows that is based on a theoretical framework for describing coupled systems. The schema combines an expanded set of traditional flowchart symbols with pictograms representing data states. The data pictograms include distributed mesh, field, and domain decomposition descriptors and spatiotemporal integration and accumulation registers. Communications pictograms include: blocking- and non-blocking point-to-point and $M \times N$ parallel data transfer; parallel data transposes; collective broadcast, scatter, gather, reduction and barrier operators. The transformation pictograms include: intergrid interpolation; spatiotemporal integral operators for accumulation of state and flux data; and weighted merging of output data from multiple source models for input to a destination model. I apply the schema to simple problems illustrating real situations in coupler design and implementation.
\end{abstract}

\section{Introduction}

Coupled systems are increasingly prevalent in computational science and engineering. Multiphysics models combine subsystem models to achieve higherfidelity simulation of the greater whole and superior solutions in their constituent processes. Multiscale models capture spatiotemporal-scale interactions by coupling separate models that operate on disparate time and length scales. Multiphysics and multiscale models share a common requirement for data exchange mechanisms that allow their constituent subsystems to compute their respective states - the coupling problem (CP) [1]. In many cases, coupled systems contain constituent subsystems possessing high levels of computational complexity that warrant parallel processing. Coupling in distributed-memory parallel environments is particularly difficult — the parallel coupling problem (PCP) [1].

Most coupled models are built by multidisciplinary teams from legacy model codes. Coupling mechanisms - or couplers - confront coupled model designers 
with complexity in choices of operation order (e.g., computation of fluxes followed by interpolation, or vice versa) and algorithms (e.g., $M \times N$ data transfer), creating the potential for uncertainty or even software bugs. This raises the question: Is there a compact way to express coupling workflow complexity that allows rapid, up-front analysis of coupler design? In this paper I propose a graphical schema for elucidating coupling workflows and employ it to explore coupler design.

Symbols and symbolic diagrams are widely used in place of words to communicate ideas. Symbol sets for diagrams are available for various disciplines, including electrical engineering, meteorology, and computer science [2]. Computational workflows traditionally are characterized by using flowcharts $3[4$ that specify control flow and processing operations in a system. Flowcharts, however, depict processing at a high level of granularity - in some cases line by line of code. If we are to use flowcharts, it must be at a coarse level of code granularity, but with sufficient detail to capture often-repeated operations such as intergrid interpolation. Data flow diagrams [5] capture the data states in various parts of a system and how information flows through a system; they do not offer detail pertaining to the transformations driving the data flows. The Unified Modeling Language (UML) 6] provides diagrams for elucidating system structure and behavior. The activity diagram depicts a series of processing steps in a system; ovals represent processing activities and their progression is represented by connecting arrows. The state diagram depicts a series of states of a system; squashed boxes represent system states, connected by labeled arrows denoting processing steps or guard expressions. UML's graphics are easy to draw, but subtle, and it's easy for nonspecialists such as computational scientists to make errors using UML [7]. None of these existing solutions suffices to cover the complete problem of documenting the transformations and data states of a coupling workflow.

I have concluded that an approach that incorporates elements of flowcharts and data flow diagrams will best suit the problem at hand. My graphical schema leverages flowcharts but augments some of its well-known symbols with pictograms that represent processing activities relevant to coupling. The schema is derived from a theoretical framework for the CP and PCP. In Section 2 I summarize this theoretical framework, defining terms for both the schema and discussion for the remainder of this paper. In Section 3 I define the schema's pictograms and drawing conventions. In Section 4 I construct schematics illustrating some commonly encountered coupling mechanisms.

\section{Coupling in Multiphysics and Multiscale Models}

Below I define terms and provide a theoretical overview of data trafficking in the $\mathrm{CP}$ and PCP; further details are available in 11. A coupled system is constructed from $N$ interacting models - or constituents $-\left\{\mathcal{C}_{1}, \ldots, \mathcal{C}_{N}\right\}$. Each model has a spatial domain $\Gamma_{i}$ plus time; intersections between spatial domains result in overlap domains $\Omega_{i j}=\Gamma_{i} \cap \Gamma_{j}$. Each model's domain boundary $\partial \Gamma_{i}$ is the intersection of its overlap domains; that is, $\partial \Gamma_{i} \equiv \cap_{j \neq i} \Omega_{i j}$. Coupling entails data 
exchange between models. Denote each model's state variables, inputs, and outputs as $\left(U_{i}, V_{i}, W_{i}\right)$. Each of these entities is a set of variables; for example, $U$ comprises the wind, temperature, pressure, and humidity fields for a simple atmosphere model. A model solves its equations of evolution on a spatial domain $\Gamma$; thus the state on the domain is a vector field resulting from the Cartesian product $U \times \Gamma$. A model's inputs (outputs) is also a vector field $V \times \partial \Gamma(W \times \partial \Gamma)$.

Models $\mathcal{C}_{i}$ and $\mathcal{C}_{j}$ are coupled if and only if at a minimum $\mathcal{C}_{i}$ provides output to (receives input from) $\mathcal{C}_{j}$. This requires $\Omega_{i j} \neq \emptyset$. In some cases the data dependency relationship is immediately obvious; that is, $W_{i} \cap V_{j} \neq \emptyset$ or $W_{j} \cap V_{i} \neq \emptyset$. In other cases, $V_{i}\left(V_{j}\right)$ is computable from $W_{j}\left(W_{i}\right)$ by a coupling transformation $\mathscr{T}_{i j}: W_{j} \rightarrow V_{i}\left(\mathscr{T}_{j i}: W_{i} \rightarrow V_{j}\right)$. Thus far we have described explicit coupling. Implicit coupling between models $\mathcal{C}_{i}$ and $\mathcal{C}_{j}$ constitutes an overlap between their respective state variables; that is, $U_{i} \cap U_{j} \neq \emptyset$. Implicit coupling requires a selfconsistent, simultaneous solution for shared-state variables using a solver $\mathscr{S}_{i j}$ (ordering of indices $i$ and $j$ is irrelevant).

Coupling events play a crucial role in the time evolution of multiscale and multiphysics systems. The time signature of the data exchanged is either instantaneous or integrated; integrated data exchanges involve the delivery of time integrated (averaged) flux (state) data from a source model to a destination model, which applies integrated fluxes incrementally during intervals between coupling events. Some coupled systems employ integrated data delivery to loosen intermodel couplings; see Section 4 of 11 for further discussion. Similarly, multiscale models may use spatially integrated data delivery to transfer information from smaller to larger length scales.

Thus far we have discussed bipartite coupling. In principle, a constituent $\mathcal{C}_{i}$ can receive the same input data from more than one model-for example, $\mathcal{C}_{j}$ and $\mathcal{C}_{k}$. In this situation, merging of data is required if there is a second-order overlap domain $\Omega_{i j k} \equiv \Gamma_{i} \cap \Gamma_{j} \cap \Gamma_{k} \neq \emptyset$ and $\mathscr{T}_{i j}$ and $\mathscr{T}_{i k}$ produce some of the same input fields among $V_{i}$. Higher-order merges may occur on higher-order overlap domains, for example, a $k-1$-way merge on the $k$ th order overlap domain $\Omega_{n_{1}, \ldots, n_{k}} \equiv \Omega_{n_{1}} \cap \cdots \cap \Omega_{n_{k}}$ for $n_{1} \neq \cdots \neq n_{k}$. Multipartite ( $k$-way) implicit coupling occurs on $\Omega_{n_{1}, \ldots, n_{k}}$ if $U_{n_{1}} \cap \cdots \cap U_{k} \neq \emptyset$ for $n_{1} \neq \cdots \neq n_{k}$; in this case a $k$-way self-consistent solver $\mathscr{S}_{n_{1}, \ldots, n_{k}}$ is required.

Coupled system models are implemented on digital computers by using numerical analysis techniques that discretize space and time. A model's gridpoints derive from a discretization $\boldsymbol{\Delta}_{i}\left(\Gamma_{i}\right)$; its boundary gridpoints are $\boldsymbol{\Delta}_{i}\left(\partial \Gamma_{i}\right)$. The state, inputs, and outputs of a numerical model $\mathcal{C}_{i}$ are its state vector $\mathbf{U}_{i} \equiv U_{i} \times \boldsymbol{\Delta}_{i}\left(\Gamma_{i}\right)$, input vector, $\mathbf{V}_{i} \equiv V_{i} \times \boldsymbol{\Delta}_{i}\left(\Gamma_{i}\right)$, and output vector $\mathbf{W}_{i} \equiv W_{i} \times \boldsymbol{\Delta}_{i}\left(\Gamma_{i}\right)$. Thus an explicit coupling transformation delivering data from $\mathcal{C}_{j}$ to $\mathcal{C}_{i}$ is $\mathscr{T}_{i j}: \mathbf{W}_{j} \rightarrow$ $\mathbf{V}_{i} ; \mathscr{T}_{i j}$ will likely comprise a field variable transformation $\mathscr{F}_{i j}$ that embodies natural-law relationships between $W_{j}$ and $V_{i}$ and a mesh transformation $\mathscr{G}_{i j}$ that maps the same variable defined on gridpoints in $\boldsymbol{\Delta}_{j}\left(\Omega_{i j}\right)$ to values defined on the gridpoints in $\boldsymbol{\Delta}_{i}\left(\Omega_{i j}\right)$. In principle, these operations do not commute; that is, $\mathscr{G}_{i j} \circ \mathscr{F}_{i j} \neq \mathscr{F}_{i j} \circ \mathscr{G}_{i j}$. This feature is a source of coupling uncertainty and a motivator for graphical representation of coupling workflows. 
Thus far we have assumed a von Neumann uniprocessor computer architecture. Introduction of concurrency complicates the CP, leading to the PCP. In a single address space, the complication is parallelization of the data transformations $\mathscr{T}_{i j}$ and any solvers required by implicit couplings. Distributed-memory architectures introduce further complications - domain decomposition of coupling data, parallel data transfer, and concurrency in model execution. Domain decomposition of data across a pool of $K$ processes is accomplished by the partitioning operator $\mathbf{P}(\cdot)$; each model will have its own domain decomposition $\mathbf{P}_{i}(\cdot)$. Thus, for model $\mathcal{C}_{i}$ resident on a pool of $K_{i}$ processes, $\mathbf{P}_{i}\left(\Gamma_{i}\right)=\left\{\gamma_{i}^{1}, \ldots \gamma_{i}^{K_{i}}\right\}$, with $\gamma_{i}^{\nu}$ the portion of $\Gamma_{i}$ resident on the $\nu$ th process; similarly, $\mathbf{P}_{i}\left(\Omega_{i j}\right)=\left\{\omega_{i j}^{1}, \ldots \omega_{i j}^{K_{i}}\right\}$, $\mathbf{P}_{i}\left(\mathbf{U}_{i}\right)=\left\{u_{i}^{1}, \ldots, u_{i}^{K_{i}}\right\}, \mathbf{P}_{i}\left(\mathbf{V}_{i}\right)=\left\{v_{i}^{1}, \ldots, v_{i}^{K_{i}}\right\}$, and $\mathbf{P}_{i}\left(\mathbf{W}_{i}\right)=\left\{w_{i}^{1}, \ldots, w_{i}^{K_{i}}\right\}$. Parallelization of $\mathscr{T}_{i j}$ and any implicit solvers requires parallel data transfer to deliver data from the source model $\mathcal{C}_{j}$ to the destination model $\mathcal{C}_{i}$; this amounts to adding a data movement operation $\mathscr{H}_{i j}$ to the mesh and field variable transformations. The data mover $\mathscr{H}_{i j}$ is a one-way parallel data (i.e., $M \times N$ ) transfer or a two-way parallel data redistribution (i.e., a transpose). Process composition is the mapping the models to pools of processes or cohorts. On a uniprocessor system, serial composition is the only option; models run in turn successively on the single resource. Multiprocessors allow further mapping strategies: parallel composition, in which models are mapped to nonoverlapping process pools and execute simultaneously on their respective cohorts; hybrid composition, which nests serial and parallel compositions to create complex process maps; and overlapping composition in which cohorts of two models partially intersect.

\section{Graphical Schema Specification}

The schema must enable users to capture the functionality present in coupling workflows. It must make clear, at a glance, design choices in terms of parallelism, process composition, and number of executable images. The pictograms must be easy to draw by hand to encourage use on paper and whiteboards. The symbol set must be sufficiently complete to cover a wide variety of coupling functions as outlined in Section 2. The schema must be extensible to allow users to create new symbols for coupling functions specific to their applications. The standard color scheme should be black and white to allow ease of sketching and to allow userdefined color coding as an additional degree of freedom in diagram construction.

Figure 1 displays the symbols and line conventions in the schema. Rectangles with rounded corners represent subsystem models. Model elements are drawn with either solid or dashed lines depending on the model's layout in the system's process composition. Ellipses represent the field, mesh, and domain decomposition data exchanged and processed during coupling. The graphical schema inherits a number of conventions from flowcharting: rectangles with sharp corners represent processing operations; a rhombus indicates a decision point; parallelograms indicate i/o operations; a triangle represents extraction of a subset of data from a data object; an upside-down triangle represents merging of multiple data objects into a single object; and a circle (upside-down house) represents 
a continuation point to a corresponding continuation point elsewhere on the same diagram (on another diagram). A burst symbol indicates parallel communications such as MPI point-to-point and collective operations. Directed arrows represent processing paths. Processing paths do not cross but may touch in the case of iterative processing. When space is tight in a diagram, one can draw processing paths appearing to cross (e.g., Figure [5), but as with flowcharts, the crossing is not significant. One may draw one of the paths with a "bump" in it to represent this skewness - a convention adopted from circuit diagrams. For parallel systems, dashed and dotted lines are employed as needed to identify processing occurring on a subset of the parent cohort; for example, differing types of lines emerging from the decision rhombus with the criterion "MyID == Root?" distinguish processing paths for root and nonroot processes.

The semantics of the schema are defined as follows. A line connecting a data (processing) element to a processing (data) element signifies an input (output) relationship to (from) the processing element. A line connecting two processing elements signifies flow of control in the direction dictated by the arrow; input data to the destination processing element is treated separately. A data element may serve as input to multiple processing elements and a processing element may have multiple outputs.

Model boxes can be annotated with pertinent information such the name of the model, as parallel or uniprocessor, parallelism mechanism, and number of processing elements. Data symbols can be annotated with descriptive information such as model name, grid name, and list of fields. Processing boxes can be annotated with the name of the algorithm embodied by the box; this is also the convention for adding new processing symbols. Continuation symbols are by definition annotated by a label indicating the continuation point; shading or blackening a continuation symbol indicates that the rest of the system in that direction of the workflow is regarded as a black box.

Multiple model boxes with dashed edges represent a serial composition (Figure 2(a)). Multiple model boxes with solid edges represent a parallel composition (Figure 2(b)). A model box with thickened solid edges indicates a separate executable image; in diagrams for which no model box of this type is present, the system is a single executable. Nesting of solid and dashed model boxes represents hybrid compositions; dashed (solid) model boxes represent serial (parallel) composition nested within a parallel (serial) composition (Figures 2(b) and (c)).

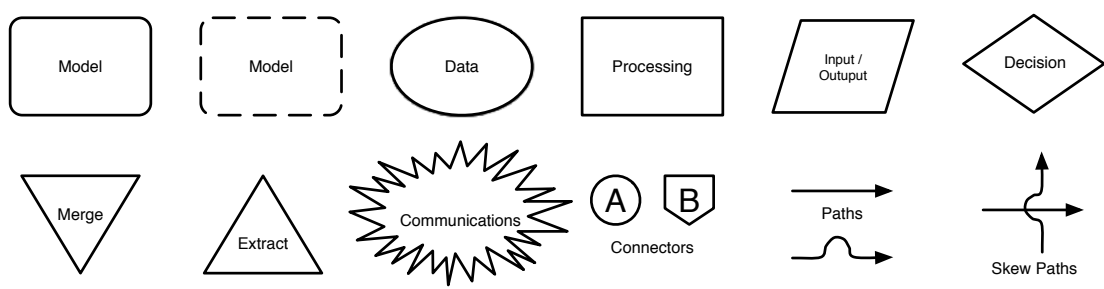

Fig. 1. Shapes of basic symbols used to diagram coupling workflows 


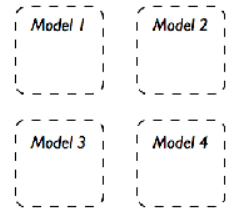

(a)

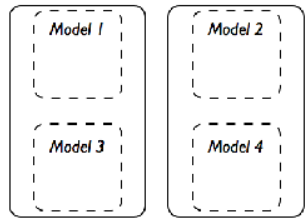

(c)

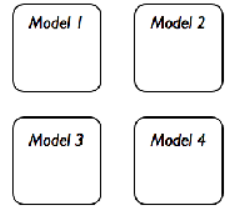

(b)

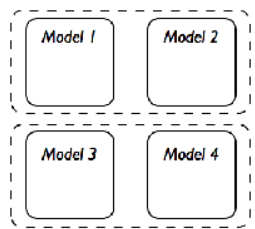

(d)

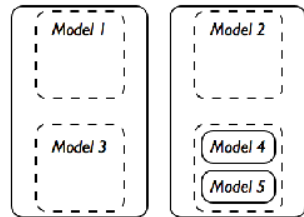

(e)

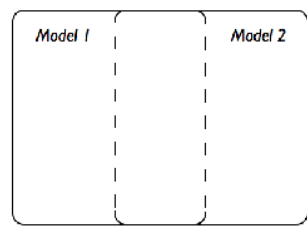

(f)

Fig. 2. Graphical conventions for representing process compositions: (a) serial composition, (b) parallel composition, (c) hybrid composition with parallel compositions embedded within a serial composition, (d) hybrid composition with serial compositions embedded within a parallel composition, (e) hybrid composition with multiple embedding levels, (f) overlapping composition

Multiple levels of nesting represent deeper levels of hybrid composition (e.g., Figure 2(e)). Intersecting boxes indicate overlapping composition (Figure 2(f)).

Coupling relies on the description of field and mesh data, and in the PCP on domain decomposition of these data. Figure 3(a) displays the pictograms for state, input, and output vectors, discretized domains and overlap regions, and domain decomposition objects. In some cases, it is convenient to incorporate field data with its resident mesh and domain decomposition in a simple object called a bundle [8], and a separate symbol is provided for this purpose. Pictograms for time- and space-integrated data objects are included. An "accumulator" is included for use either in place of the integrated field objects or to represent results of global reductions (e.g., MPI_REDUCE()) across a process pool. Not all of the data pictograms used in this paper are shown in Figure 3 (a). Pictograms for state, coupling. and mesh data partitioned across a process pool are labeled by using notation from Section $2,\{\mathbf{u}, \mathbf{v}, \mathbf{w}, \boldsymbol{\Delta}(\gamma), \boldsymbol{\Delta}(\omega)\}$ in place of $\{\mathbf{U}, \mathbf{V}, \mathbf{W}, \boldsymbol{\Delta}(\Gamma), \boldsymbol{\Delta}(\Omega)\}$. Also, for ease of hand-drawing of schematics, the bold-face notation on pictograms may be replaced with vector symbols; for example, $\vec{U}$ or $\underset{\sim}{U}$ in place of $\mathbf{U}$. Integrated data may also be represented by using triangular brackets- $\langle\mathbf{W}\rangle,\langle\mathbf{w}\rangle$, and so on.

Figure 3(b) displays the core set of pictograms used to depict intercomponent data movers $\mathscr{H}_{i j}$ and communications operations commonly used in couplers, including blocking- and nonblocking point-to-point messaging and $M \times N$ transfers, parallel data transposes, broadcasts, scatters, gathers, and synchronization. Other communications pictograms are created by annotating an empty burst symbol-for example, a global reduction. 


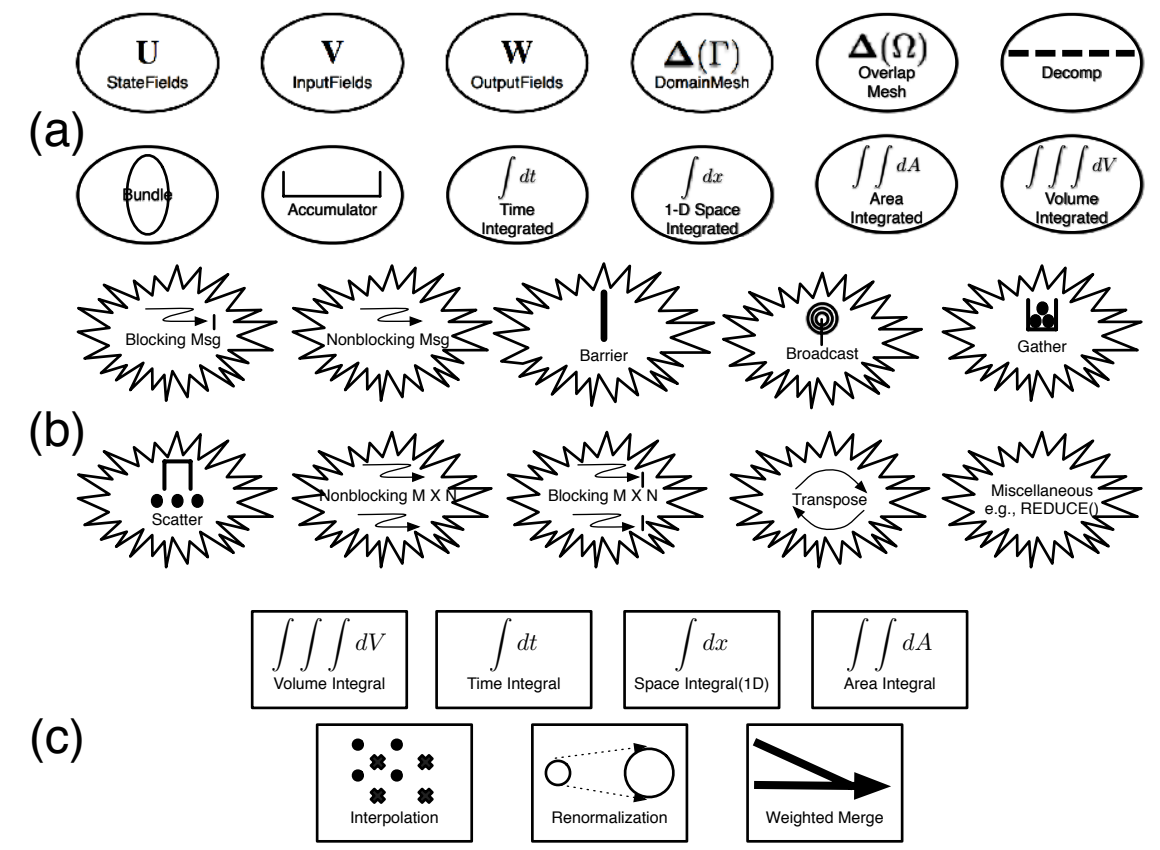

Fig. 3. Detailed pictograms for representing (a) data, (b) communications, and (c) data transformation

Figure 3](c) displays the core set of data transformation pictograms, including intergrid interpolation, temporal and spatial integration, renormalization to enforce conservation of flux integrals, and weighted merging.

\section{Examples}

A common coupled systems problem is intermodel data transfer. Figures 4 and 5 show the model-coupler communications patterns for versions 2 and 3 of the Community Climate System Model, respectively; see 8 for further details. Figure 4 depicts the communications between a model employing hybrid MPI/OpenMP parallelism and a coupler that is solely OpenMP parallel but possesses one MPI process. The models are in parallel composition, each implemented in a separate executable. Distributed output data $\mathbf{w}$ is gathered from the atmosphere's cohort to its root, yielding W. Nonroot processes wait in a barrier until the root finishes communicating with the coupler. A single blocking MPI message containing $\mathbf{W}$ is sent to the coupler, which receives it as input $\mathbf{V}$. The coupler in turn posts a single blocking message to the atmosphere and continues processing upon completion. The atmosphere receives this message as $\mathbf{V}$, and scatters the message to its processes, yielding v. Figure 5 depicts parallelized $M \times N$ data transfer between two separate executables, both possessing multiple MPI processes. The atmosphere sends its distributed output $\mathbf{w}$ in parallel to 
the coupler. Once the parallel send has completed, the atmosphere receives its distributed input $\mathbf{v}$ from the coupler. All communications are blocking overall; that is, an MPI_Waitall() is invoked to ensure that the model and coupler evolve together.

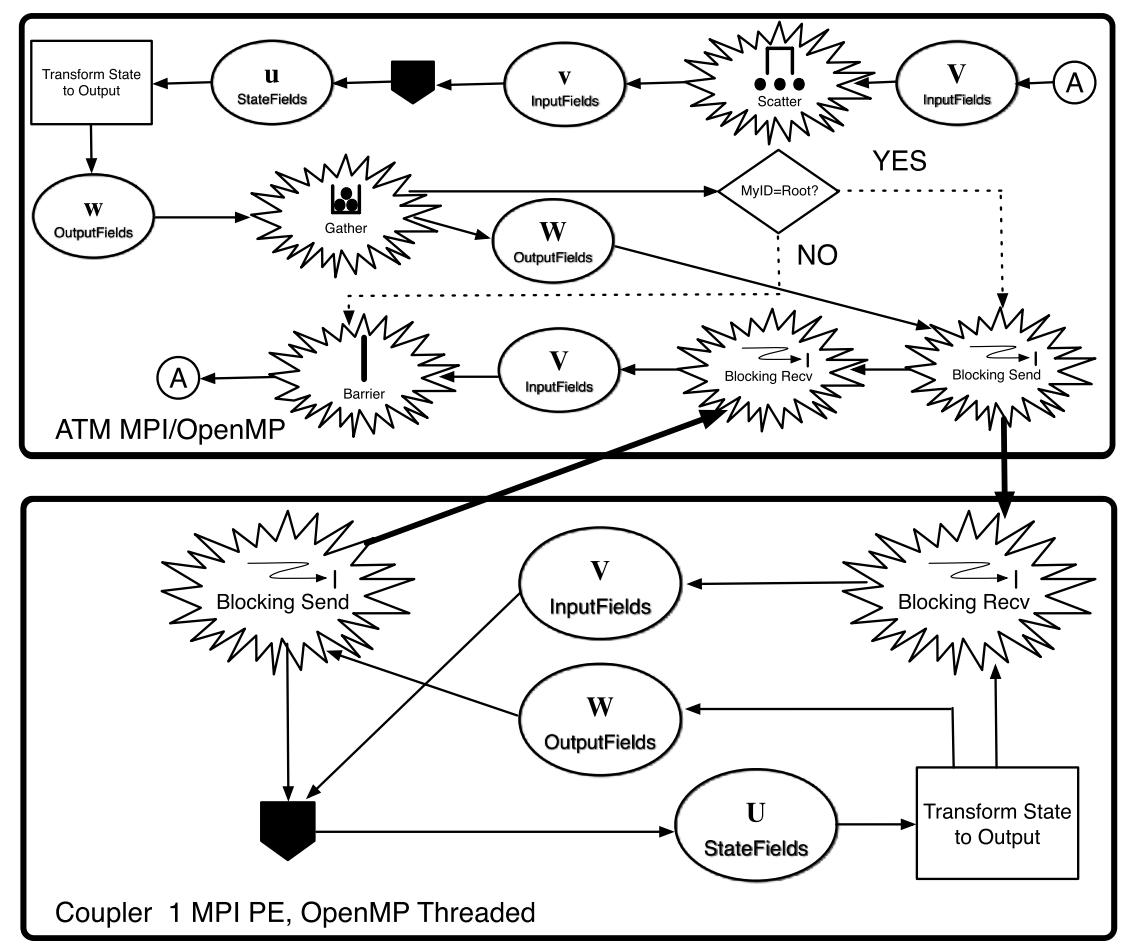

Fig. 4. Coupling workflow between two models featuring serialized communications

Three key types of data transformations used in coupling are shown in Figure 6 Figure6(a) depicts integrated data delivery processing. A model takes distributed instantaneous output $\mathbf{w}$ and integrates it with respect to time, accumulating fluxes and averaging state data. This process is performed over a coupling cycle period, and the decision box determines whether the model should continue or pause to couple to the rest of the system. Figure 6(b) shows a scheme for enforcing conservation of interfacial fluxes under interpolation. Distributed input data $\mathbf{v}$ defined on Grid 1 are received and integrated across the two- dimensional boundary. The data are then interpolated to yield input defined on Grid 2; they are integrated on this grid over the boundary. The values of $\mathbf{v}$, defined on Grids 1 and 2, together with their respective integrals, are then passed through a renormalization function, which computes the ratio of the integrals obtained on Grids 1 and 2 and uses their ratio to rescale the values of $\mathbf{v}$ residing on Grid 2, thus conserving global flux integrals across the boundary. 


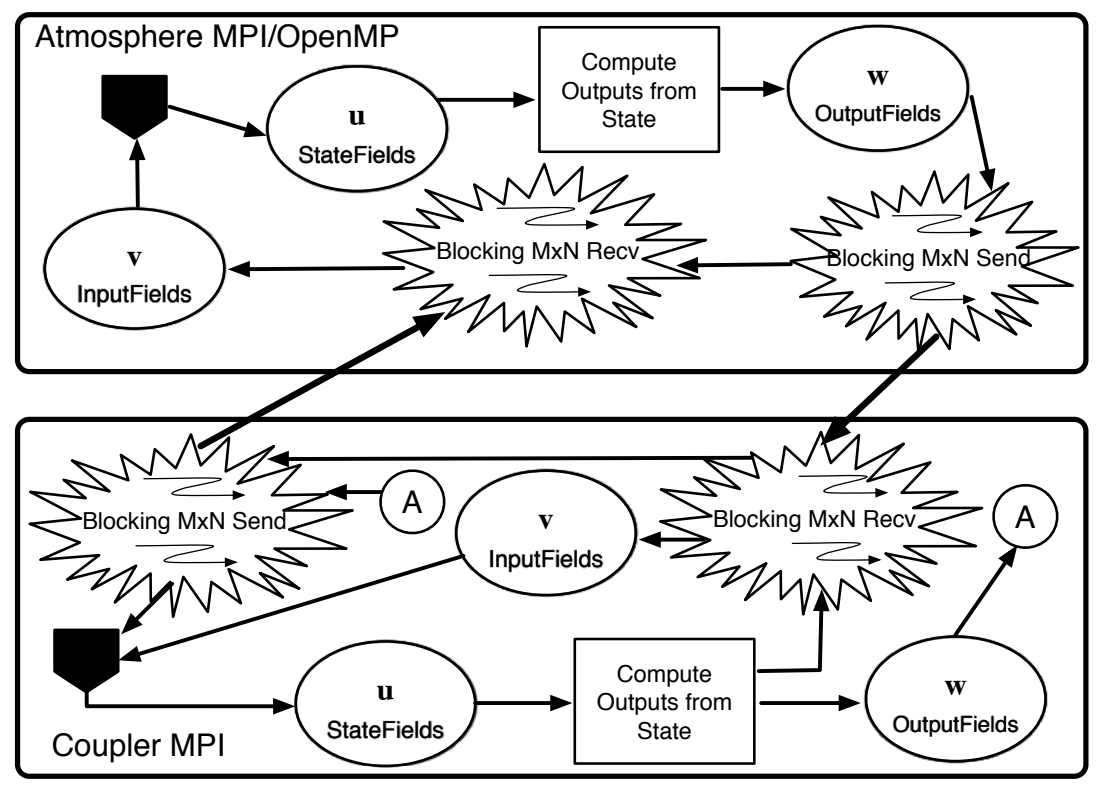

Fig. 5. Coupling workflow between two models featuring $M \times N$ transfer

(a)

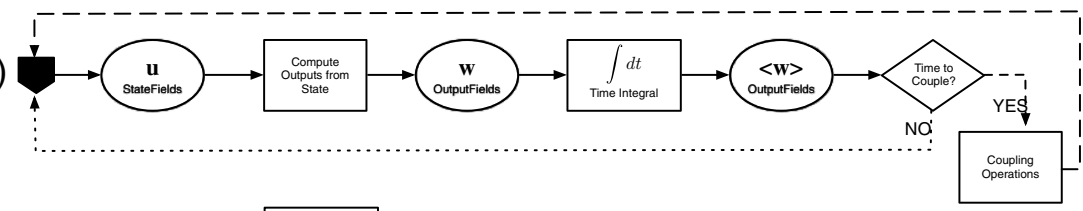

(b)

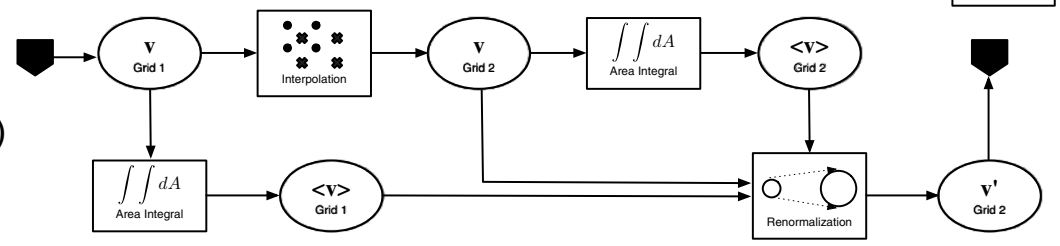

(c)

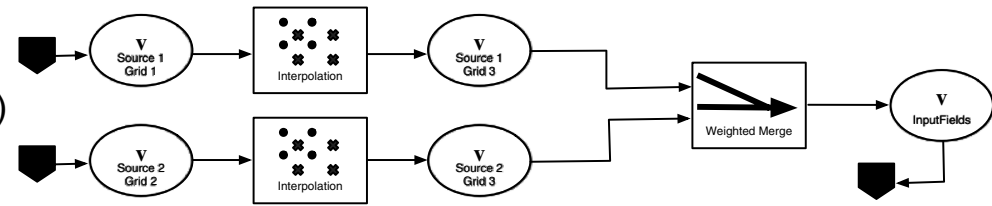

Fig. 6. Coupling workflows for (a) time-integrated data delivery, (b) flux-conserving interpolation, and (c) merging of inputs 


\section{Conclusions}

I have proposed a graphical schema for describing intermodel data coupling that combines control and data flow. The schema is derived from a theoretical framework for describing intermodel coupling. The schema is rich enough to describe a wide variety of intermodel coupling situations, and user-extensible. I have employed the schema to depict commonly-encountered coupling workflows.

Associating the schema with software entities - for example, a generic coupling infrastructure toolkit such as the Model Coupling Toolkit (MCT) [9]—will enable graphical program specification for coupler mechanisms. This will require mappings between the schema and MCT's classes and methods, combined with code generation infrastructure, and is an exciting area for future investigation.

Acknowledgments. This work was supported by the US Department of Energy's Scientific Discovery through Advanced Computing program. Argonne National Laboratory is operated for the DOE by UChicago Argonne LLC under contract DE-AC02-06CH11357. I thank the Department of Theoretical Physics/ RSPhysSE at ANU for hosting me as a visiting fellow.

\section{References}

1. Larson, J.W.: Ten organising principles for coupling in multiphysics and multiscale models. ANZIAM Journal (accepted) (2008)

2. Dreyfuss, H.S.: Symbol Sourcebook: An Authoritative Guide to International Graphic Symbols. McGraw-Hill, New York (1972)

3. ANSI: Standard flowchart symbols and their use in information processing (X3.5). Technical report, American National Standards Institute, New York (1970)

4. ISO: Information processing-documentation symbols and conventions for data, program, and system flowcharts, program network charts and system resources charts. Technical report, International Organization for Standardization, Geneva (1985)

5. Yourdon, E.: Structured analysis wiki (2009), http://www.yourdon.com/strucanalysis/

6. OMG: Unified Modeling Language web site (2009), http://www.uml.org/

7. Bell, E.: Death by UML fever. ACM Queue 2(1), 72-80 (2004)

8. Craig, A.P., Kaufmann, B., Jacob, R., Bettge, T., Larson, J., Ong, E., Ding, C., He, H.: cpl6: The new extensible high-performance parallel coupler for the community climate system model. Int. J. High Perf. Comp. App. 19(3), 309-327 (2005)

9. Larson, J., Jacob, R., Ong, E.: The model coupling toolkit: A new fortran90 toolkit for building multi-physics parallel coupled models. Int. J. High Perf. Comp. App. 19(3), 277-292 (2005) 